\title{
ZU PÉTER LENGYELS COBBLESTONE
}

\author{
MIRKO DOMBROWSKY \\ Universität zu Köln, Köln, \\ Deutschland
}

Im Berlin der zwanziger Jahre war mitunter von Asphaltliteratur die Rede. Damit bezeichnete man - nicht ganz ohne Wertung - jene Texte vornehmlich aus jüdischer Tradition, die sich den Themen Großstadt und Zivilisation zuwendeten. Diese Diskurspolitik diente nicht zuletzt der Abgrenzung von „wahrer“ Dichtung oder „Weltliteratur“, wobei man in beiden Fällen gerne an Goethe dachte. Das vielleicht prominenteste Beispiel von „Zivilisationsliteratur“ aus dieser Zeit ist Alfred Döblins Roman Berlin Alexanderplatz, welcher freilich trotz der Absage Döblins an das klassisch-bürgerliche Bildungsideal selbst bald Einzug in die sogenannte Weltliteratur hielt. 1988 nun veröffentlicht der ungarische Autor Péter Lengyel den Großstadtroman Macskakö, in dem wiederum der Straßenbelag eine Rolle zu spielen scheint. Der Titel der englischen Übersetzung von 1993 lautet Cobblestone.* Schauplatz der Haupthandlung ist Budapest während der ungarischen Millenniumsfeier im Jahr 1896. Da liegt es nahe, an Musils Mann ohne Eigenschaften zu erinnern, in dem die andere Metropole der habsburgerischen Doppelmonarchie keine zwanzig Jahre später ebenfalls das Epizentrum einer patriotischen Jubiläumsveranstaltung ist (welche sich hier freilich nur wortreich ankündigt). Welchen Aufschluß geben diese literarischen Assoziationen?

Ein Blick auf die Romananfänge läßt vermuten, daß auch Lengyel durch die Wahl des Sujets Großstadt dem Gang der Zivilisation nachspürt. Es geht hinaus auf die Straße. Dort spielt sich das moderne Leben ab, und dort sind es jeweils die neuen Verkehrsmittel, die als Signifikanten rasanter Veränderungen Verunsicherung und Krisenerfahrung mit sich bringen.

Er stand vor dem Tor des Tegeler Gefängnisses und war frei. [...] Er ließ Elektrische auf Elektrische vorbeifahren, drückte den Rücken an die rote Mauer und ging nicht. Der Aufseher am Tor spazierte einige Male an ihm vorbei, zeigte ihm seine Bahn, er ging nicht. (Berlin Alexanderplatz: S. 8)

* Eine deutsche Übersetzung liegt nicht vor.

Hungarian Studies 14/1 (2000)

0236-6568/2000/\$5.00 @ 2000 Akadémiai Kiadó, Budapest 
Schon einen Augenblick vorher war etwas aus der Reihe gesprungen, eine quer schlagende Bewegung; etwas hatte sich gedreht, war seitwärts gerutscht, ein schwerer, jäh gebremster Lastwagen war es [...] Die Dame fühlte etwas Unangenehmes in der Herz-Magengrube, das sie berechtigt war für Mitleid zu halten; es war ein unentschlossenes, lähmendes Gefühl. (Der Mann ohne Eigenschaften: S. 10f.)

It was on the afternoon of this day that back home, on the Pest side of our city, the first automobile put-putted past the thieves' dens of Cemetery Road [...]. Eventually it would reach the traces of the lesser branch of the Danube [...]; where, as it turned, a guardian of the law, with the feathergrass in his hat, attempted to arrest this suspicious contraption speeding by without the aid of visible horses. (Cobblestone: S. 9)

Auf vergleichbare Weise taucht somit eine zentrale Frage der Moderne in allen drei Romanen bereits auf den ersten Seiten auf: es geht um Bewahrung oder Wiederherstellung von Ordnung in der Unüberschaubarkeit der Metropole. Durch die Episode mit dem ersten Auto wird in Cobblestone vorweggenommen, daß hier den Hütern des Gesetzes diese Aufgabe zukommt. So ist auch allen Texten gemein, daß das Durchbrechen der Ordnung die Form des Verbrechens annimmt. Begegnen uns in Berlin Alexanderplatz und im Mann ohne Eigenschaften die Frauenmörder Biberkopf und Moosbrugger, so haben wir es in Clobblestone mit einer Gang von Edelganoven zu tun, die sich auf dem Kopfsteinpflaster Budapests einen kriminologischen und nicht zuletzt technologischen Wettstreit mit dem Special Investigator Dr. Dajka liefern. Allein durch die in Europa völlig neue Fingerabdrucktechnik kann letzterer dem Kopf der Bande auf die Schliche kommen. Einen existentiellen oder gar philosophischen Hintergrund läßt das Safeknacken an sich jedoch vermissen. Ebensowenig wird die Tat an moralischen Maßstäben gemessen. Bei Clobblestone handelt es sich durch und durch um einen Krimi, der durchaus Spannung aus der Frage bezieht, ob die Gangster zum Schluß geschnappt werden. Insofern scheint es etwas hochgestochen, wenn im Untertitel von einem ,philosophical mystery for the Millennium“ die Rede ist. Zwar liefert der Roman eine detailgetreue Milieustudie der Budapester Unterwelt. Er porträtiert "life's richest variety along the cobblestone streets of our capital city“ (Cobblestone: S. 436). Dabei geht es aber offensichtlich weniger darum, die moralische Verunsicherung der sich rasant wandelnden Gesellschaft der Jahrhundertwende zu reflektieren. Die Frage, ob dann aber der Hinweis auf die Asphaltliteratur und ihren zivilisationskritischen Impetus gerechtfertigt ist, soll zunächst zurückgestellt werden.

Ein hervorstechendes formales Merkmal des Romans ist das Inhaltsverzeichnis. Dort werden die Kapitelüberschriften, die stets schon ein thematisches Ele- 
ment des Kapitels ankündingen, durch eine ausführliche Liste von Stichwörtern ergänzt. Diese - wenn auch kryptische - Vorausschau erinnert an die Tradition der Synopsen, die auf den vorbürgerlichen Roman zurückgeht. Bei diesem Typ Roman handelt es sich stets auch um eine Art Nachschlagewerk, denn das Wichtigste wird durch die moritätenhafte Ankündigung des Inhalts bereits vorweggenommen. Das ermöglicht eine andere Art des Lesens, ein wissendes, enzyklopädisches Lesen, das weniger auf Einfühlung als auf Reflexion setzt. Man kann sich anhand des Inhaltsverzeichnisses aussuchen, zu welchem Thema man sich ,informieren“ will. Döblin und Musil, und freilich auch Brecht, greifen auf dieses Verfahren als Mittel der Illusionsdurchbrechung zurück, getreu Musils Motto, daß es im 20. Jahrhundert nicht um die Bildung der Charaktere im Sinne der realistischen Tradition, sondern um die Bildung der Ideen gehe. Es wird kein Zufall sein, daß Lengyel sich in diese Tradition des An- und Vorausdeutens stellt - und sie zugleich ironisiert. Denn das Stichwortverzeichnis stellt sich dem Leser als ein Puzzle dar, aus dem er erst nach sorgfältigen Lesen schlau wird. Zugleich deutet sich durch die verwirrende Vorwegnahme des Rohmaterials in den Überschriften an, daß auch in diesem Roman der Reflexion eine besondere Rolle zukommt.

Tatsächlich handelt es sich bei Cobblestone um eine ausgesprochen selbstreflexive Konstruktion. Lengyel flicht in die Haupterzählung einen Metadiskurs in Form von Kommentaren und Reflexionen eines fiktionalen Alter Egos ein. Die Einschübe nehmen die Form von einzelnen Sätzen bis zu ganzen Kapiteln an und sind stets durch Klammern markiert. Diese Rahmenhandlung fingiert eine narrative Vermitlungssituation, in der ein Schriftsteller seiner Tochter, die er stets mit „Du“ anredet, über etliche Jahre hinweg die Geschichte des Juwelenraubs aus der Perspektive des Kopfs der Bande erzählt. Diese Vorgehensweise des Autors erfüllt mehrere Funktionen, durch welche der historische Krimi sich dann doch als sehr nachdenkliche Auseinandersetzung mit den zivilisatorischen Entwicklungen dieses Jahrhunderts entpuppt:

Erstens wird die Zuhörerin darüber aufgeklärt, welchen Namenswechseln und Veränderungen die Schauplätze des Geschehens unter kommunistischer Herrschaft unterzogen wurden. Damit werden diese zu realen Orten, die einen (unglücklichen) geschichtlichen Prozeß bezeugen. Nur in wenigen Fällen kann der Erzähler wie folgt feststellen, daß die alten Namen als Bestandteil des kulturellen Gedächtnisses der Bevölkerung nicht durch die irgendwelcher Funktionäre ersetzt wurden: „For you: still Izabella, this lucky one has survived.“ (Cobblestone: S. 134) Interessanterweise widmet der Erzähler bei seiner Skizzierung der sich wandelnden Topographie der Stadt der Straßenoberfläche große Aufmerksamkeit. Natürlich müssen im Lauf der Zeit die Pflastersteine an unzähligen Stellen dem Asphalt weichen. Ironisch beschreibt er ihn als ,the new miracle surface [...] easy to keep marvelously clean" (Cobblestone: S. 101.) Der Siegeszug des neuen Straßenmaterials wird immer wieder zynisch kommentiert. Ausnahmen bestätigen die 
Regel: „These trees are here to stay, making this one spot where the asphalt, and all its pimps, will not triumph." (Cobblestone: S. 134). Durch die leitmotivische Beschreibung der Straßenoberfläche rückt Lengyel die zentrale Bedeutung der materiellen Gegebenheiten für das moderne Leben in der Stadt in den Vordergrund. Im Gegensatz zum Pflasterstein ist der Asphalt Kennzeichen von Sterilität und Kälte. Es stellt sich heraus, daß Lengyel doch - in einem sehr wörtlichen Sinne - Asphaltliteratur geschrieben hat.

So liefert der Ich-Erzähler der Rahmenhandlung zweitens Eindrücke des zeitgenössischen Lebens auf den asphaltierten Plätzen und Straßen Budapests. Cobblestone ist vor allem auch ein Roman über die Geschichte einer Stadt und funktioniert über den Kontrast zwischen der atmosphärischen, abenteuerlichen und weltläufigen Metropole der Jahrhundertwende und der Bescheidenheit der Verhältnisse nach vierzig Jahren Kommunismus. Welch ein Unterschied, ob man im dunklen Umhang durch das nächtliche Budapest streift und das Verbrechen des Jahrhunderts vollbringt oder ob man sich sorgt, weil die Tochter Probleme im Leichtathletikverein hat. Die Safeknacker übernachten in den prachtvollen Hotels an der Riviera. Bei einer Pragreise kommt der Erzähler ein einer Schule unter, dabei hätte die Tochter so gerne einmal im Hotel übernachtet. Diese Gegensätze bewirken eine melancholische Grundstimmung. So bleiben auch gelegentliche Seitenhiebe auf das kommunistische System nicht aus. Zynisch erinnert der Erzähler an die Marxismusvorlesungen, in those fine days when the Piarists were no longer allowed to drug the workers' suspectible minds with the opiate of religion" (Cobblestone: S. 143).

Drittens reflektiert der Erzähler die Bedingungen und Möglichkeiten des Erzählens. Dabei wird deutlicher, weshalb er einer historischen Bestandsaufnahme als Folie einen Krimi unterlegt:

Cobblestone birgt in sich auf vielfache Weise den Proze $\beta$ des Registrierens, Ordnens und Verstehens von Informationen. Da sind zum einen die Ermittlungen Dajkas. Seine Aufgabe besteht darin, Fakten zu sammeln und zu einem sinnvollen Ganzen zusammenzusetzen. Der Rekonstruktion des Tathergangs hat er sich mit Leib und Seele verschrieben. Um einen vergleichbaren Vorgang handelt es sich jedoch auch beim Erzählvorgang des Ich-Erzählers der 1896-Handlung selbst, der nicht nur die Ereignisse, die seinem Wissensstand entsprechen, sondern auch die der Gegenseite zu einer kohärenten Erzählung zusammenfügen will. Seine Glaubwürdigkeit als Erzähler gewinnt er nur teilweise dadurch zurück, daß im letzten Teil „The Way it Was“ im Chinese-Box-Verfahren der pensionierte Dajka und sein Nachfolger dem Erzähler die Ereignisse aus ihrer Sicht mitteilen. Daß in ihrer Erzählung der Ich-Erzähler, der ihnen gerade zuhört, bei einem Schiffsuntergang ums Leben kommt, ist schon herrlich vertrackt. Über diese erzähltechnischen Schwierigkeiten reflektiert wiederum der fiktive Autor, der Ich-Erzähler der 
Gegenwartshandlung, und setzt sich dabei grundsäztlich mit den Problemen des Romanschreibens auseinander:

In seiner langen Tradition wurde der Roman immer wieder als Abbild des Lebens in seiner ganzen Komplexität definiert. Für Friedrich Schlegel sollte der Roman die „Enzyklopädie eines gesamten Lebens“ darstellen, also bei $A$ anfangen und bei $Z$ aufhören. Aber was ist das $A$ und was das $Z$ im Leben, und was kommt in der Mitte? „Well then, what are you going to put into your book" wird der Schriftsteller von seiner Tochter gefragt. Seine zutreffende Antwort lautet: "That is what we're going to have to sort out. [...] Perhaps the whole book is going to be about that." (Cobblestone: S. 155f.) So lauten die Leitfragen dieses metafiktionalen Diskurses, den der Erzähler zwischen den Klammern führt ,what to include in our story?" und ,at what point to begin?" (Cobblestone: S. 192). Wie beantwortet er sie? Lawrence Sterne geht schon recht weit zurück und fängt nicht bei der Geburt, sondern bei der Zeugung Tristam Shandys an. Der Erzähler von Cobblestone treibt es auf die Spitze und beginnt mit dem Urknall. Parallel zum narrativen Strang der Haupterzählung beginnt er eine zweite Erzählung und überfliegt die gesamte Menschheitsgeschichte. Er zeigt uns, wie er unter Einsatz seines wichtigsten Instruments, ,the zoom lens“ (Cobblestone: S. 301), die Einstellung verkleinert, bis er Budapest im 1896 im Sucher hat und der Stand der Dinge dort eingeholt ist. Dies weckt den Eindruck, daß Fokussierung stets nur ein Zufallsprodukt ist. Man hätte ebenso gut jeden anderen Ort zu jeder anderen Zeit heranzoomen können. Die Motivation, so lernen wir, bleibt hingegen immer gleich: „to arrest the unstoppable flow of time, to recall a piece of it, to tear it out and store it away for all of us..." (Cobblestone: S. 32).

Informationsselektion ist aber nicht nur ein poetologisches Problem des Romans. In Cobblestone taucht es auf allen Ebenen auf. Der Kriminologe Dajka muß entscheiden, was für den Fall relevant ist und dann die Teile des Mosaiks zusammenfügen, ,as one continuous narrative“ (Cobblestone: S. 427). Die Geschichte, die er sich zusammenreimt, muß stimmig und glaubhaft sein. So ist sein Problem tatsächlich ein narratologisches. „Detective work is like writing fiction.“ (Cobblestone: S. 395) Der zentrale Unterschied besteht darin, daß der Autor „in advance“, der Ermittler jedoch ,in hindsight“ (Cobblestone: S. 453) weiß, was passiert. Was anderes tut aber ein Vater, der versucht, seinem Kind die Welt zu erklären (die er nicht versteht)? Sein übertriebener Versuch, alles in eine groß Erzählung der Menschheitsgeschichte zu integrieren und auch wirklich mit dem Anfang anzufangen, wird konterkariert durch seine zerstreuten Notizen über den Alltag, die angesichts von Themen wie Aids, Obdachlosigkeit, Alkoholismus, Jugendgewalt, Zensur, die also angesichts einer „horrendous new world“ (Cobblestone: S. 211). Ausdruck seiner Orientierungslosigkeit sind. Mit Hayden White ließe sich sagen, daß Geschichten und Geschichtsschreibung wohl dem 
fundamentalen Wunsch entspringen, der Offenheit des Lebens etwas entgegenzusetzen, „to put an image of continuity, coherency, and meaning in place of our fantasies of emptiness, need, and frustrated desire that inhibit our nightmares the destructive power of time“" (White: S. 15). In Cobblestone setzt Lengyel den WeltErklärungsnöten des Vaters Dajkas unermüdliches "striving for completeness“ (Cobblestone: S. 197) entgegen und reflektiert damit, daß das Mosaik des Lebens nur in der Fiktion (nahezu) vervollständigt werden kann. „Life is always more multi-dimensional than theories of literary esthetics." (Cobblestone: S. 428.)

Cobblestone stellt sich somit in die Reihe der Romane der Gegenwart, die sich als historiographische Metafiktion bezeichnen lassen und die die Bedeutung narrativer Strukturen für die Verarbeitung nicht-fiktionalen Geschehens reflektieren. Dies tut auch Lengyel, explizit, indem er sein fiktionales Alter Ego Überlegungen über die Schwierigkeiten des Erzählens anstellen läßt, und implizit, indem er für seine Geschichte des vergangenen Jahrhunderts die Form des Krimis wählt, eine narrative Form, die nach Geschlossenheit drängt und die im Kontrast zum Diskurs des Vaters um so mehr die Unwägbarkeiten des Alltags sowie die Absurditäten der Menschheitsgeschichte zu Tage legt. Dieser Kontrast funktioniert auch über das plastisch vor Augen geführte Gegensatzpaar von Pflasterstein und Asphalt. Nicht zuletzt soll die Geschichte des Jahrhundert-Verbrechens jedoch (die Tochter) in ihren Bann ziehen und ein anschauliches Porträt der Weltstadt Budapest aus vergangenen, vorsozialistischen Tagen liefern. 